\title{
Common motor patterns of asymmetrical and symmetrical bipedal gaits
}

\author{
Germán Pequera ${ }^{\text {Corresp., } 1,2}$, Ignacio Ramírez Paulino ${ }^{3}$, Carlo M. Biancardi ${ }^{2}$ \\ 1 Ingeniería Biológica, CENUR Litoral Norte, Universidad de la República, Paysandú, Uruguay \\ 2 Biomechanics Lab., Dept. de Ciencias Biológicas, CENUR Litoral Norte,, Universidad de la República, Paysandú, Uruguay \\ 3 Inst. de Ingeniería Eléctrica, Fac. de Ingeniería, Universidad de la República, Montevideo, Uruguay \\ Corresponding Author: Germán Pequera \\ Email address: gpequera@cup.edu.uy
}

Background. Synergy modules have been used to describe activation of lower limb muscles during locomotion and hence to understand how the system controls movement. Walking and running have been shown shared synergy patterns suggesting common motor control of both symmetrical gaits. Unilateral skipping, an equivalent gait to the quadrupedal gallop in humans, has been defined as the third locomotion paradigm but the use by humans is limited due to its high metabolic cost. Synergies in skipping have been little investigated. In particular, to the best of our knowledge, the joint study of both trailing and leading limbs has never been addressed before.

Research question. How are organized muscle activation patterns in unilateral skipping? Are they organized in the same way that in symmetrical gaits? If yes, which are the muscle activation patterns in skipping that make it a different gait to walking or running? In the present research, we investigate if there are shared control strategies for all gaits in locomotion. Addressing these questions in terms of muscle synergies could suggest possible determinants of the scarce use of unilateral skipping in humans.

Methods. Electromyographic data of fourteen bilateral muscles were collected from volunteers while performing walking, running and unilateral skipping on a treadmill. Also, spatiotemporal gait parameters were computed from 3D kinematics. The modular composition and activation timing extracted by nonnegative matrix factorization were analyzed to detect similarities and differences among symmetrical gaits and unilateral skipping.

Results. Synergy modules showed high similarity throughout the different gaits and between trailing and leading limbs during unilateral skipping. The synergy associated with the propulsion force operated by calf muscles was anticipated in bouncing gaits. Temporal features of synergies in the leading leg were very similar to those observed for running. The different role of trailing and leading legs in unilateral skipping was reflected by the different timing in two modules. Activation for weight acceptance was anticipated and extended in the trailing leg, preparing the body for landing impact after the flight phase. A different behaviour was detected in the leading leg, which only deals with a pendular weight transference.

Significance. The evidence gathered in this work supports the hypothesis of shared modules among symmetrical and asymmetrical gaits, suggesting a common motor control despite of the infrequent use of unilateral skipping in humans. Unilateral skipping results from phase-shifted activation of similar 
muscular groups used in symmetrical gaits, without the need for new muscular groups. The high and anticipated muscle activation in the trailing leg for landing could be the key distinctive event of unilateral skipping. 


\section{Common motor patterns of asymmetrical and symmetrical bipedal gaits}

Germán Pequera $^{1,2}$, Ignacio Ramirez Paulino ${ }^{3}$, Carlo M. Biancardi ${ }^{1}$

1 Biomechanics Lab., Dept. de Ciencias Biológicas, CENUR Litoral Norte, Universidad de la República, Uruguay.

2 Ingeniería Biológica, CENUR Litoral Norte, Universidad de la República, Uruguay.

3 Inst. de Ingeniería Eléctrica, Fac. de Ingeniería, Universidad de la República, Uruguay.

11

12

13

14

15

16

17

Corresponding Author:

Germán Pequera

Ingeniería Biológica

19 CENUR Litoral Norte

20 Florida 1065

2160000 Paysandú

22 Uruguay

23 Email address: gpequera@cup.edu.uy

24

25

26

27

28

29

30

31

32

33

34

35

36

37

38

39

40

41

42 


\section{Abstract}

44 Background. Synergy modules have been used to describe activation of lower limb muscles 45 during locomotion and hence to understand how the system controls movement. Walking and symmetrical gaits. Unilateral skipping, an equivalent gait to the quadrupedal gallop in humans, has been defined as the third locomotion paradigm but the use by humans is limited due to its high metabolic cost. Synergies in skipping have been little investigated. In particular, to the best of our knowledge, the joint study of both trailing and leading limbs has never been addressed before.

Research question. How are organized muscle activation patterns in unilateral skipping? Are they organized in the same way that in symmetrical gaits? If yes, which are the muscle activation patterns in skipping that make it a different gait to walking or running? In the present research, we investigate if there are shared control strategies for all gaits in locomotion. Addressing these questions in terms of muscle synergies could suggest possible determinants of the scarce use of unilateral skipping in humans.

Methods. Electromyographic data of fourteen bilateral muscles were collected from volunteers while performing walking, running and unilateral skipping on a treadmill. Also, spatiotemporal gait parameters were computed from 3D kinematics. The modular composition and activation timing extracted by non-negative matrix factorization were analyzed to detect similarities and differences among symmetrical gaits and unilateral skipping.

Results. Synergy modules showed high similarity throughout the different gaits and between trailing and leading limbs during unilateral skipping. The synergy associated with the propulsion force operated by calf muscles was anticipated in bouncing gaits. Temporal features of synergies in the leading leg were very similar to those observed for running. The different role of trailing and leading legs in unilateral skipping was reflected by the different timing in two modules. Activation for weight acceptance was anticipated and extended in the trailing leg, preparing the body for landing impact after the flight phase. A different behaviour was detected in the leading leg, which only deals with a pendular weight transference.

Significance. The evidence gathered in this work supports the hypothesis of shared modules among symmetrical and asymmetrical gaits, suggesting a common motor control despite of the infrequent use of unilateral skipping in humans. Unilateral skipping results from phase-shifted activation of similar muscular groups used in symmetrical gaits, without the need for new muscular groups. The high and anticipated muscle activation in the trailing leg for landing could be the key distinctive event of unilateral skipping.

82

8




\section{Introduction}

89

90

91

92

93

94

95

96

97

98

99

100

101

102

103

104

105

106

107

108

109

110

111

112

113

114

115

116

117

118

119

120

121

122

123

124

125

126

127

128

129

130

131

132

Although walking and running are the most used gaits, the repertoire of bipedal locomotion also includes skipping, a bipedal gait equivalent to gallop (Saibene \& Minetti, 2003). Unlike walking and running, skipping is an asymmetric gait, where one foot behaves differently from the other. We can distinguish a trailing limb (the first of a pair to strike the ground) from a leading limb (Hildebrand, 1977). Also, we identify two unilateral skipping forms, equivalents to the quadrupedal right- and left-leading transverse gallop, and a bilateral form, equivalent to the quadrupedal rotary gallop (Minetti, 1998; Biancardi \& Minetti, 2012).

The mechanical model of skipping-gallop is a double pogo-stick, with a step vaulting phase (similar to walking) followed by a bouncing phase (as occurs in running). These features are distinctive enough for some authors to refer to skipping as the "third paradigm of locomotion" (Minetti, 1998; Saibene \& Minetti, 2003). Skipping is energetically demanding with respect to its quadrupedal homologous (Minetti et al., 1999, Biancardi \& Minetti, 2012), or compared to walking and running (Minetti, 1998; Ackermann \& Van den Bogert, 2010; Minetti, Pavei \& Biancardi, 2012; Pavei, Biancardi \& Minetti, 2015), and therefore seldom used by humans. This studies commonly have been addressed metabolic and mechanical parameters in different conditions of speed and gravity to explain the high metabolic power developed by humans during this gait. However, very few studies have performed muscle activation analysis to investigate how similar are the activation patterns outputs between this gait and common symmetrical gaits (Ivanenko et al. (2008)).

Muscle activation patterns during locomotion have been described by means of muscle synergies (or motor modules) (Cappellini et al, 2006; Ting \& McKay, 2007). Each synergy represents the activity of the co-activated muscles triggered by a single control signal. This scenario suggests the existence of a control mode in a low-dimensional space, rather than a specific control command for each muscle. Although the role and existence of such modules are still questioned (Tresch \& Jarc, 2009), the muscle synergy hypothesis provides a good interpretation of the neural control strategy for activating muscles during locomotion (Lacquaniti, Ivanenko \& Zago, 2012; Ting et al., 2015; Yokoyama et al., 2019). Synergy identification has been done based on electromyography signals (EMG) from muscles of the limbs; these groups of signals were then decomposed by applying various computational methods (Tresch, Cheung \& d'Avella, 2006). Cappellini et al. (2006) showed that, while walking or running on a straight path at a different controlled speed, the lower limbs muscles group in similar five modules; the only difference between walking and running was the temporal activation of one of the modules. Being walking and running symmetric, the authors assumed that the synergies would have been the same in the two limbs, and thus measured the electromyographic signals on the muscles of only one limb (Cappellini et al., 2006). The same approach was followed by other authors, which confirmed the shared basic synergy modules at different inclinations in walking (Saito et al., 2018a), and in running (Saito et al., 2018b); the same was observed in walking vs. cycling (Barroso, 2014). Different authors associated each synergy module to a particular step phase (Capellini et al., 2006; Neptune, Clark, \& Kautz, 2009; Santuz et al., 2018b). However, to the best of our knowledge, no synergy studies have been conducted considering both the leading and trailing limbs in asymmetric gaits like unilateral skipping. In this sense, studying the unilateral skipping asymmetries in terms of muscular synergies could help to identify common underlying control mechanisms, shared among gaits.

Peer] reviewing PDF | (2020:10:54165:2:0:NEW 15 Jul 2021) 
133 Our main hypothesis was that the synergy modules should be shared by both symmetric and

134 asymmetric gaits, with even timing differences between trailing and leading limbs of unilateral

135

136

137

138

139

140

141

142

143

144

145

146

147

148

149

150

151

152

153

154 skipping. Based on this, the following working hypotheses have been defined:

- Walking, running and unilateral skipping presents shared muscle synergies, with differences in their activation timing;

- The activation timings of the trailing and the leading limbs of skipping differ;

- According to the mechanical model of skipping as a combination of pendulum and spring-mass, the activation timings of the trailing and leading legs may be more similar to walking or running; alternatively, the activation of one limb may be similar to that of walking while the other may be similar to that of running.

\section{Materials \& Methods}

\section{Participants}

Fourteen healthy men took part in the experiment (age: $25.3 \pm 3.7$ years, weight: $78.15 \pm 13.8$ $\mathrm{kg}$, height: $1.76 \pm 0.09 \mathrm{~m}$ ). We excluded subjects with muscular pain, cardiovascular or neuromuscular diseases.

All the participants signed a written informed consent according to the Declaration of Helsinki. The project was approved by the Ethics Committee of the Centro Universitario Regional Litoral Norte, Universidad de la República (Exp. No 311170-000521-18).

\section{Experimental protocol}

The protocol was completed in a single session. At the beginning, subjects warmed up and practiced unilateral skipping overground and on the treadmill until they were familiar with the task. Afterwards, they were asked to walk, run and skip unilaterally at controlled speeds on a treadmill. Ten trials of four minutes were conducted: walking at $0.83,1.11,1.39,1.81 \mathrm{~m} . \mathrm{s}^{-1}$; running at $1.81,2.50$ and $3.06 \mathrm{~m} . \mathrm{s}^{-1}$; and unilateral skipping at $1.39,1.81$ and $2.50 \mathrm{~m} . \mathrm{s}^{-1}$. These speeds were chosen based on previous experiences (Minetti, Pavei \& Biancardi, 2012; Pavei, Biancardi, \& Minetti, 2015), with some overlap so that different gaits could be compared at the same velocities.

The order of the speeds were randomized within each gait condition. In order to avoid muscle fatigue, there was a three minute rest between trials.

\section{Data Acquisition}

Electromyographic signals were bilaterally recorded from Gluteus Medius (GluM), Bíceps Femoris (long head, $B F$ ), Rectus Femoris $(R F)$, Vastus Medialis $(V M)$, Tibialis Anterior $(T A)$, Gastrocnemius Medialis $(G M)$ and Soleus $(S o l)$ during the last 90 seconds of each trial. The electrode placement in each muscle was realized following the SENIAM protocols (Stegeman and Hermens, 2007). This muscle set was chosen based on the analysis of muscle synergies locomotion studies when less than ten muscles by leg were measured (Clark et al., 2010; Barroso et al., 2014; Pérez-Nombela et al, 2017; Shuman et al, 2019). Signals were sampled at 2 $\mathrm{kHz}$ by a Delsys Trigno EMG system (Boston, MA, USA), bandpass filtered between $20 \mathrm{~Hz}$ to $400 \mathrm{~Hz}$, and amplified (x10000). Each Trigno unit includes a single differential EMG sensor synchronized to a triaxial accelerometer.

In the same time window, kinematic data were recorded by an eight-camera MOCAP system (Vicon, Oxford Metrics), at $100 \mathrm{~Hz}$. Eighteen markers were located on the main joint centers in 
179

180

181

182

183

184

185

186

187

188

189

190

191

192

193

194

195

196

197

198

199

200

| 201

202

203

204

205

206

207

208

209

210

211

212

213

214

215

216

217

218

219

220

221

222

223

order to reconstruct the 3D movement with a reliable biomechanics model composed of eleven segments (Pavei et al., 2017).

\section{Kinematic analysis}

Accelerometric signals from the $T A$ sensor were used to identify heel strikes (Oliveira et al., 2016). Stride frequency $(F s)$ was computed as the reciprocal of the cycle duration, while the stride length $(L s)$ was obtained by dividing $F s$ into the progression speed. The duty factor $(D F)$ (Alexander, 2003) that is, the fraction of the total cycle in which a given foot is on the ground, was computed by identifying the times of landing and take-off of the heel and fifth metacarpal markers (Pavei, Biancardi \& Minetti, 2015). Flight times $\left(F_{T}\right)$ during skipping and running were calculated using the same markers.

The body Center Of Mass (COM) positions were determined as the weighted mean of the centers of mass of the segments, which were in turn determined using anthropometric Dempster tables (Winter, 2003). The trajectory of the COM 3D position within a stride, in local coordinates (as displacing on a treadmill), would describe a closed curve (Minetti, 2009; Minetti, Cisotti and Mian, 2011). In symmetrical gaits, the trajectory exhibits a double loop, each one representing one step, including the stance phase of one limb and the swing phase of the other (Minetti et al., 2011).

\section{EMG processing}

The dominant leg of each subject was assessed before the experimental session (Van Melick et al., 2017). The EMG of the dominant limb during walking and running, and those of both limbs during_unilateral skipping were processed. By spontaneous choice of each participant, the dominant leg was used by all participants as trailing leg during unilateral skipping. EMG were full-wave rectified and low-pass filtered (Butterworth, 4th order, cutoff frequency $=10 \mathrm{~Hz}$ ). In each trial, 20 strides were normalized in amplitude by the peak activation signal of the gait cycle, resampled at $1 \%$ of the gait cycle and concatenated, resulting in a matrix $E M G_{o}(m \times t)$, where $m$ $=7$ (the number of muscles) and $t=2000$ (number of samples). A non-negative matrix factorization $(N N M F)$ algorithm was applied to the matrix in order to identify the muscle synergies (Lee \& Seung, 1999; Ting \& Macpherson, 2005). Under this model, the $E M G_{o}$ matrix can be decomposed (factorized) as:

$$
E M G_{o}=W \times C+R=E M G_{r}+R
$$

The number of synergies identified by the model is given by the number of columns of the matrix $W(m \times n)$, which coincides with the number of rows in the matrix $C(n \times t)$. The i-th synergy is described by the i-th column of $W$, which specifies the relative weight of the $m$ muscles within the synergy, and the corresponding i-th row of $C$, which describes the activation pattern of the synergy across time. $E M G_{r}$ is the reconstructed EMG matrix while the matrix $R(m$ $\mathrm{x} t$ ) is considered a residual error of the model.

To reduce the effect of local minima, the $N N M F$ algorithm was executed 40 times with random initial conditions and the factorization with the lowest squared reconstruction error was selected. To identify the number of synergies, $N N M F$ was applied on bootstrapped EMG signal considering a different number of synergies each time, from 1 to 7. $E M G_{o}$ of five randomly chosen subjects in each condition was concatenated $\left(E M G_{b}\right)$. This procedure was repeated 20 
224

225

226

227

228

229

230

231

232

233

234

235

236

237

238

239

240

241

242

243

244

245

246

247

248

249

250

251

252

253

254

255

256

257

258

259

260

261

262

263

264

265

times in each trial to estimate confidence intervals of the variance accounted for (VAF) for each number of synergy. The VAF defined as:

$$
V A F=1-\frac{\left\|E M G_{b}-E M G_{r b}\right\|_{F}^{2}}{\left\|E M G_{b}\right\|_{F} 2}
$$

where $F$ represents the Frobenious norm and $E M G_{r b}$ represents $E M G_{b}$ reconstruction from $N N M F$. VAF vs synergy number plots were used to determine the appropriate number of synergies for each condition. The point at which the VAF curve shows a considerable slope change is determined as the appropriate synergy number. As Cheung et al (2005) report, the slope of the VAF plot usually decreases gradually with the synergy number, creating difficulties to estimate, by visual inspection, the number of synergies correctly. For hence, the number of synergies was identified from a linear regression method already used by several authors (Cheung et al., 2005; d'Avella et al., 2006; Santuz et al., 2018b). This method identifies the smallest number of synergies such that a linear fit of the VAF curve, from 1 to 7 , had a residual mean square error of less than $1 \times 10^{-4}$.

The weightings were sorted using the maximal cosine similarity (cosim) (Hagio \& Kouzaki, 2014; Hagio, Fukuda \& Kouzaki, 2015; Banks et al., 2017). Given two weight vectors $a$ and $b$, their cosim is given by

is the cosine of the angle between the two weight vectors.

cosim was calculated between weightings modules of an arbitrary subject and the rest of subjects employing an iterative process. We defined the pair of modules with the highest cosim value as the same group. (Hagio \& Kouzaki, 2014; Torres-Oviedo \& Ting, 2007). cosim was also used to measure the similarity between motor modules across different gaits; values of cosim above 0.6 commonly indicate similarity between motor modules (d'Avella, Saltiel \& Bizzi, 2003).

The temporal features of module activation were evaluated using a center of activation ( $\mathrm{CoA})$. CoA represents the center of mass of the circular distribution of the temporal components in polar coordinates $\left(0 \leq \theta_{j} \leq 2 \pi\right)$ (Santuz et al., 2018a; MacLellan, 2017), and is given by $C o A=$ $\tan ^{-1}\left(\frac{B}{A}\right)$ where

$$
A=\sum_{j=1}^{100}\left(\cos \theta_{j} \times C_{j}\right) \text { and } B=\sum_{j=1}^{100}\left(\sin \theta_{j} \times C_{j}\right)
$$

where $C j$ is the amplitude of the activation pattern in sample $j$.

\section{Statistical analysis}

Differences in spatiotemporal parameters and in the number of synergies between gaits were evaluated using a one-way ANOVA, or Kruskal-Wallis test if Shapiro-Wilk tests on the residuals report non-normality. T-tests (or Wilcoxon rank-sum test, in case of non-normality) with Bonferroni correction were applied to reduce the risk of Type I errors in multiple hypothesis testing scenarios. One sample t-test was realized on cosim values in each condition comparison to check if synergy pairs were significantly larger than the 0.6 (similarity threshold). The $C o A$ of 
266

267

268

269

270

271

272

273

274

275

276

277

278

279

280

281

282

283

284

285

286

287

288

289

290

291

292

293

294

295

296

297

298

299

300

301

302

303

304

305

306

307

308

309

310

311

different gaits for fixed speeds were compared using the Watson-Williams test for circular data. Statistical significance was assessed at 0.05 .

\section{Results}

The displayed results show only comparisons at $1.81 \mathrm{~m} . \mathrm{s}^{-1}$, where the three gaits are present. Comparisons at $1.39 \mathrm{~m} . \mathrm{s}^{-1}$ and at $2.5 \mathrm{~m} . \mathrm{s}^{-1}$ can be found in supplementary material.

\section{Gait parameters}

Table 1 resumes the spatio-temporal parameters of all the analysed gaits. $L s$ and Fs presents statistical differences between all gaits (all p-values equal or less than 0.001).

The $D F$ of the trailing and leading limbs of unilateral skipping was significantly different at $1.81 \mathrm{~m} \cdot \mathrm{s}^{-1} . D F$ during unilateral skipping in the leading leg did not displayed statistical differences with running (posthocs p-values near 1 at $1.81 \mathrm{~m} \cdot \mathrm{s}^{-1}$ ). $D F$ in walking showed differences with all gaits and legs. Significant $p$-values less than 0.01 were reported between walking and both unilateral skipping legs, when multiple comparisons were realized at $1.81 \mathrm{~m} \cdot \mathrm{s}^{-1}$. Table 1 reveals also the unilateral skipping and running flight times as fraction of stride times. $F T$ in unilateral skipping were on average longer than in running showing statistical significance $(\mathrm{p}<0.001)$ at $1.81 \mathrm{~m} . \mathrm{s}^{-1}$. Longer FT imply greater average vertical ground reaction force during the contact phase of unilateral skipping, as pointed out by Minetti et al. (2012).

\section{EMG envelopes}

During walking, muscles of Triceps Surae were active at mid-stance, $R F$ and Vas were active finalizing swing phase and at the beginning of stance phase, Tib was active mostly during the swing; while $B F$ and GluM show activation during the late swing and at early stance (Figure 1). In running, the extensor muscles present EMG peak activation at the early stance phase while $T i b, B F$ and $G l u M$ presents similar activation patterns than walking.

During skipping we observed different muscle activation patterns between both legs, mostly in $T i b, R F$ and GluM muscles (Figure 1). Tib shows peak activation at the early swing in the trailing leg, while in the leading leg this muscle seems to activate at toe-off and late swing. In $R F$ can be seen a double peak in the EMG envelope of the leading leg; one at early stance and other at the toe-off. In trailing leg $R F$ is not activated at toe-off. GluM present activation at the early stance and a slow but sustained increment of activation during swing phase in trailing leg, distinct to leading leg where GluM present a similar pattern to running with activation at the early stance and late swing. The rest of the muscles present similar activation patterns shape but with anticipated activation when trailing leg is compared with leading leg in skipping.

\section{Muscle synergies}

Figure 2 shows the number of synergies required in each gait at $1.81 \mathrm{~m} . \mathrm{s}^{-1}$. When the number of synergies were evaluated in different gaits, Kruskal-Wallis test did not identify differences $(p=$ 0.068). Around 3 synergies, in average, were necessary to meet the reconstruction quality criteria used in this work in all conditions (Figure 2).

As Figure 2 points out, the mean of the number of synergies was greater than 3 in some conditions. In order to compare all gaits and speeds, 4 synergies were used in all subsequent analyses (Clark et al., 2010; Barroso et al, 2014; Pérez-Nombela et al, 2017; Shuman et al, 
312 2019). The modules were composed basically by the same muscles in all gaits. Module 1

313 included GluM, RF and $V L$; Module 2 included Triceps Surae muscles; Module 3 covered Tibial 314 muscle; Module 4 comprised $B F$ (Figure 3). When muscle weightings were compared across

315 gaits, similarity was observed across all gaits and legs (Figure 4): in most comparisons, 3 of the 4 316 modules presented highly similar mean values in all conditions; however, synergy 4 appears to

317 be specific to each locomotor pattern. On the other hand, the trailing leg in unilateral skipping 318 displayed only one shared synergy when compared to walking at $1.81 \mathrm{~m} \cdot \mathrm{s}^{-1}$.

319

320

321

322

323

324

325

326

327

328

329

330

331

332

333

334

335

336

337

338

339

340

341

342

343 Discussion

Synergy temporal patterns

Figure 5 shows the mean $C O A$ of each module across gaits at $1.81 \mathrm{~m} . \mathrm{s}^{-1}$. Some significant differences can be observed. Synergy 1 for unilateral skipping trailing leg shows late $C o A$ and significant differences with all gaits (Figure 5). CoA in walking, running and leading leg in unilateral skipping did not present statistical differences between them for this synergy. For the propulsion synergy (module 2), we can identify 3 groups of $\mathrm{CoA}$ classified by statistical differences (Figure 5). One group includes an anticipated activation of synergy 2 in trailing leg, another group includes running and leading leg in skipping $C o A$ and the third group shows a delayed $C O A$ in synergy 2 for walking (Figure 5). Synergy 3 shape two groups based in statistical test application. One anticipated group of $C O A \mathrm{~s}$ composed by both legs of unilateral skipping and other group composed by symmetrical gaits showing a belated CoA (Figure 5). Synergy 4 did not present statistical differences between gaits (Figure 5).

\section{Center of mass trajectory}

In Figure 6 are plotted the trajectories of the $C O M$ during three single strides, taken as examples of walking, running and unilateral skipping. According to the kinematics of the $C O M$ movement, the lower parts of the loops represent the phases of heel strike and acceptance of the weight for the stance limb, followed by the propulsion phase at the moment that the COM is rising. The colors highlight the effects of the activation of the first two synergy modules on the stance limb. In unilateral skipping, the lower vertices of the triangular loop represent, as indicated in the figure, the trailing and leading stance phases. A clear asymmetry can be seen of the synergistic activation during unilateral skipping (Figure 6).

344

345

346

347

\section{Gait parameters}

During unilateral skipping, the trailing and leading legs play different roles, the former being more dedicated to vertical braking and forward propulsion, and the latter vice versa, to forward braking and vertical propulsion (Fiers et al., 2013). Considering the high average vertical force observed during the stance phase of unilateral skipping, in accordance with Minetti et al. (2012), and the ability of the muscles to absorb negative work, it is reasonable to expect that the $D F$ of the trailing limb will be lower than that of the leading limb, as detected by Fiers et al. (2013). Our results confirm this trend (Table 1).

The $D F$ of the leading leg was not significantly different from that of running at the same speed. The $F_{S}$ of running was on average $1.32 \mathrm{~Hz}$, near the optimal value of $1.4 \mathrm{~Hz}$ (Snyder \& Farley, 2011). With respect to running, unilateral skipping displayed significantly higher $F_{S}$ and significantly lower $L_{S}$ at the same speed (Table 1). This confirms the previously observed pattern in (Minetti, Pavei \& Biancardi, 2012; Pavei, Biancardi \& Minetti, 2015; Fiers et al., 2013). 
358 Higher $F_{S}$ can lead to higher energy expenditure, which is one of the characteristics of unilateral 359 skipping with respect to running (Snyder \& Farley, 2011; Pavei, Biancardi \& Minetti, 2015).

360 One reason for that could be related to the necessity to increase the muscle shortening velocities, 361 in order to achieve higher $F_{S}$ (Lindstedt et al., 1985).

362

363

364

365

366

\section{Muscle synergies}

To the best of our knowledge, this was the first simultaneous evaluation of synergies of the trailing and leading limbs in unilateral skipping. Different works on muscle synergies during locomotion reported 4-5 synergies to reconstruct the original EMG signals (Capellini et al, 2006 Yokoyama, et al 2016; Santuz et al, 2018a), while our work shows an average of around 3 in all conditions (Figure 3). This result could be explained by two reasons. First, we recorded a smaller set of muscles ( 7 by leg) than other works (Capellini et al, 2006; Yokoyama, et al 2016; Santuz et al, 2018a). Some authors demonstrated that the number of muscles measured affects the VAF values, overestimating them when the number of EMG signals diminish (Steele, Tresch \& Perreault, 2013). In fact, Pérez-Nombela and collaborators (2017) obtained a number of synergies comparable to our work, measuring seven muscles by side in the control group. The other reason is that there is no agreement about a standard method to estimate the number of synergies from the VAF curve (Turpin, Uriac \& Dalleau, 2021), and this fact creates difficulties to compare results associated with this parameter.

According to Santuz et al. (2018a) and Capellini et al. (2006), the same number of synergies can describe walking and running. Our results confirm those conclusions, adding unilateral skipping (both trailing and leading limbs) to the gait cluster (Figure 3). The number of synergies has been interpreted as a locomotor output complexity indicator, where a greater number of modules reflects more complexity (Clark et al, 2010). Based on these results we suggest that unilateral skipping does not imply differences in motor complexity when compared with common symmetrical gaits. For hence, the disuse of unilateral skipping would be not determined by different complexity in the task execution.

According to our first hypothesis, we found the number and the composition of the motor modules to be similar across the repertoire of human locomotion, including both legs during the asymmetrical unilateral skipping gait as Figure 3 and Figure 4 shows. Synergy modules from 1 to 3 were shared in all gaits and legs, while the fourth module revealed lower values of similarity Figure 4. Yet, the $B F$ was always the principal component of Module 4 (Figure 4). In unilateral skipping, both legs displayed weighting coefficients similar to each other and to the symmetrical gaits motor modules (Figure 4). These similarities across the different locomotor patterns suggest a common control strategy for symmetric and asymmetric gaits in humans. The above idea was originally proposed by Whitall and Caldwell (1992) when they observed similar intralimb coordination, however under different interlimb coordination, where unilateral skipping was compared to running. So, an important finding of this work is the evidence of an shared control scheme between all human gaits. Similarities between the module weighting coefficients of walking and running was already observed in previous investigations (Capellini et al., 2006); their results underlined higher correlations when the motor modules of the lower limbs muscles were compared in walking and running. Conversely, lower correlations appeared when including the upper limbs and trunk muscles in the analysis. Some authors detected differences among the synergies of different gaits and speeds, attributing this difference to the methodology used (Yokoyama et al., 2016). Recently, a walking and running neuromusculoskeletal model has been developed based on parameters of the muscle synergy 
404 hypothesis, confirming that both gaits can be produced by the same set of modules with different 405 activation timing (Aoi et al., 2019). According to our results, it should be possible to make this 406 model work for unilateral skipping as well.

407 Unilateral skipping is homologous to the transverse gallop of quadrupeds, which is their most 408 economical gait at high speeds. The fact that synergies were shared in all three gaits, including 409 unilateral skipping, highlights the idea of the synergies like phylogenetically conserved 410 structures, discussed by other authors (Dewolf et al, 2020). However, despite preserved neural 411 circuitry in evolution of locomotion, the efficiency of gait patterns varies in function of the 412 different biomechanical and anatomical characteristics of the species, and can be a strong 413 selective factor for or against the employment of gait patterns.

414

\section{Synergy temporal patterns}

As a summary we can say that walking and running are only differentiated by an anticipated activation of the Triceps Surae muscles; leading leg during unilateral skipping only is differentiated of running by anticipated activation of synergy 3 and $C o A$ in synergy 3 is able to distibguish symmetrical and asymmetrical gaits. The temporal pattern of trailing leg during unilateral skipping appears very different to those of symmetrical gaits ( 3 out of $4 \mathrm{CoA}$ were different in the majority of the comparisons). The above results indicate that the movements of the trailing leg in unilateral skipping was not similar to those of walking or running. On the other hand, our comparison of trailing and leading legs in unilateral skipping yielded 2 different $\operatorname{Co} A$ across the gait cycle, thus indicating some similarities of the temporal activation of both limbs. The first module, which includes the knee extensors ( $R F$ and $V L)$ and the hip extensor (GluM), was always activated at the beginning of the stance phase, first to absorb the negative work due to landing (weight acceptance) and then to start rising the centre of mass (Figure 5). This is in accordance with previous results on walking (Neptune, Clark \& Kautz, 2009; Clark et al., 2010; Mehrabi, Schwartz \& Steele, 2019), and running (Santuz et al., 2018b; Oliveira et al., 2016). In unilateral skipping, almost all the impact is absorbed by the trailing leg. Again, this agrees with the high extensor moments at the hip joint in trailing legs during flight time and foot contact that have been reported (Walter \& Carrier, 2007); this may explain why the activation of the weight acceptance in the trailing leg during unilateral skipping was significantly anticipated with respect to the leading leg and to the other gaits (synergy 1 in trailing leg, Figure 5). In this sense, we suggest that the effort invested by synergy 1 in the trailing leg to reduce impact in landing could be one of the keys of the high metabolic cost of transport reported by other authors (Minetti, 1998; Pavei, Biancardi \& Minetti, 2015).

Our results confirm the temporal pattern described in previous papers for symmetrical gaits (Capellini et al., 2006; Saito et al., 2018a; Saito et al., 2018b; Neptune, Clark \& Kautz, 2009; Santuz et al., 2018b; Oliveira et al., 2016; Clark et al., 2010; Mehrabi, Schwartz \& Steele, 2019). According to Cappellini et al. (2006), the difference between running and walking was the time shift of the activation component of the second synergy, associated to the plantar flexor muscles. The pattern of activation of this module in the leading limb during unilateral skipping was analogous to running (Figure 5). Therefore, we may consider this behavior of the plantar flexor module as a distinctive characteristic between bouncing versus vaulting gaits. The anticipated activation was partially due to the shorter stance phase of bouncing gait with respect to walking: the calf muscles would provide forward propulsion and contribute to body support (Neptune, Clark \& Kautz, 2009). Ivanenko et al. (2008) identified two distinct activations of the same set of muscles within a bilateral skipping cycle; they analysed EMG recorded from the 
450 right leg during bilateral skipping, where the same limb acts alternatively as trailing and leading, 451 and this could explain the two peaks in an averaged cycle (Ivanenko et al., 2008).

452 The ankle dorsiflexor tibialis anterior was included in the third synergy. Its $C o A$ extended during 453 the swing phase of walking (Neptune, Clark \& Kautz, 2009) and running (Oliveira et al., 2016). 454 In unilateral skipping, this module was anticipated to the very early swing phase, just after the 455 toe-off. The fourth synergy was associated with the thigh extension, during the late swing and 456 just before landing (Walking: Neptune et al., 2009; Clark et al., 2010; Running: Oliveira et al., 457 2016; Santuz et al., 2018b). The activation timing of this synergy was shared among gaits. 458 According to our second hypothesis, during unilateral skipping we found significant differences in $C o A$ between the skipping trailing and leading legs in synergy 1 (weight acceptance) and synergy 2 (propulsion) (Figure 5). The behavior of the two limbs is clearly different during the stance phase, but shares the same synergy pattern on the swing phase (C3 and $\mathrm{C} 4$, Figure 5). The results in Figure 5 refute our third hypothesis by showing very different activation patterns in the trailing leg than in walking, although the behavior of the leading leg in unilateral skipping is very similar to running.

\section{Center of mass trajectory}

The effect of the synergic muscle work on the COM trayectory during weight acceptance and propulsion is shown by the 3D trajectory of the COM in Figure 6 (Minetti, Cisotti \& Mian, 2011). In walking, the propulsive phase accompanies the rise of the $C O M$ up to its highest point, while the weight acceptance begins with the heel strike and continues as the body switches from double to single support (Figure 6A). In running, the propulsive phase ends with the take-off, while the COM continues its rise during the ballistic phase (Figure 6B).

In unilateral skipping, the already mentioned asymmetry is evident (Figure 6C). The anticipated weight acceptance phase prepares the trailing leg to support the impact after the flight phase. The two propulsion phases are very different, as in one case is a quasi-pendular weight transfer from trailing to leading while pushing forward, while the leading propulsion must push the up for takeoff.

\section{Conclusions}

According to our hypothesis (i) and (ii), muscle synergies are shared across the repertoire of human locomotion and activation timing of trailing and leading limbs were different. Therefore, in healthy people, unilateral skipping is not determined by different synergistic structure, but by adjustments in the basic motor patterns used in symmetrical gaits. The opportunity that the muscles have to activate within a stride cycle is what determines the biomechanical locomotor pattern of unilateral skipping, and not the modular structure. Our results, which at least partially refute our third hypothesis, support the idea of a mechanical paradigm different from those of walking and running. After having analyzed and comparing electromyographic data we can conclude that all human gaits present underlying features of muscle activation that suggest common neural control of locomotion.

\section{Acknowledgements}

The authors acknowledge Dr. Renata Bona and Dr. Artur Bonezi for helping during the data collection. The authors also thanks Martín Arévalo for IT support; A. Saito and an anonymous referee for their critics and comments, which help to improve the paper. 
496

497

498

499

500

501

502

503

504

505

506

507

508

509

510

511

512

513

514

515

516

517

518

519

520

521

522

523

524

525

526

527

528

529

530

531

532

533

534

535

536

537

538

539

540

541

\section{References}

Ackermann, M., \& Van den Bogert, A. J. (2010). Optimality principles for model-based prediction of human gait. Journal of biomechanics, 43(6), 1055-1060. DOI: https://doi.org/10.1016/j.jbiomech.2009.12.012

Alexander, R.McN. Principle of animal locomotion. Princeton University Press, 2003

Aoi, S., Ohashi, T., Bamba, R., Fujiki, S., Tamura, D., Funato, T., Senda, K., Ivanenko Y. \& Tsuchiya, K. (2019). Neuromusculoskeletal model that walks and runs across a speed range with a few motor control parameter changes based on the muscle synergy hypothesis. Scientific reports, 9(1), 1-13. DOI: https://doi.org/10.1038/s41598-01837460-3

Banks, C. L., Pai, M. M., McGuirk, T. E., Fregly, B. J., \& Patten, C. (2017). Methodological choices in muscle synergy analysis impact differentiation of physiological characteristics following stroke. Frontiers in Computational Neuroscience, 11, 78. DOI: https://doi.org/10.3389/fncom.2017.00078

Barroso, F. O., Torricelli, D., Moreno, J. C., Taylor, J., Gomez-Soriano, J., Bravo-Esteban, E., Piazza, S., Santos, C. \& Pons, J. L. (2014). Shared muscle synergies in human walking and cycling. Journal of neurophysiology, 112(8), 1984-1998. DOI: https://doi.org/10.1152/jn.00220.2014

Biancardi, C. M., \& Minetti, A. E. (2012). Biomechanical determinants of transverse and rotary gallop in cursorial mammals. Journal of Experimental Biology, 215(23), 4144-4156. DOI: $10.1242 /$ jeb.073031

Cappellini, G., Ivanenko, Y. P., Poppele, R. E., \& Lacquaniti, F. (2006). Motor patterns in human walking and running. Journal of neurophysiology, 95(6), 3426-3437. DOI: https://doi.org/10.1152/jn.00081.2006

Cheung, V. C., d'Avella, A., Tresch, M. C., \& Bizzi, E. (2005). Central and sensory contributions to the activation and organization of muscle synergies during natural motor behaviors. Journal of Neuroscience, 25(27), 6419-6434.

Clark, D. J., Ting, L. H., Zajac, F. E., Neptune, R. R., \& Kautz, S. A. (2010). Merging of healthy motor modules predicts reduced locomotor performance and muscle coordination complexity post-stroke. Journal of neurophysiology, 103(2), 844-857. DOI: https://doi.org/10.1152/jn.00825.2009

d'Avella, A., Saltiel, P., \& Bizzi, E. (2003). Combinations of muscle synergies in the construction of a natural motor behavior. Nature neuroscience, 6(3), 300-308. DOI: https://doi.org/10.1038/nn1010

d'Avella, A., Portone, A., Fernandez, L., \& Lacquaniti, F. (2006). Control of fast-reaching movements by muscle synergy combinations. Journal of Neuroscience, 26(30), 77917810.

Dewolf, A. H., Sylos-Labini, F., Cappellini, G., Lacquaniti, F., \& Ivanenko, Y. (2020). Emergence of Different Gaits in Infancy: Relationship Between Developing Neural Circuitries and Changing Biomechanics. Frontiers in Bioengineering and Biotechnology, 8, 473. DOI: https://doi.org/10.3389/fbioe.2020.00473

Fiers, P., De Clercq, D., Segers, V., \& Aerts, P. (2013). Biomechanics of human bipedal gallop: asymmetry dictates leg function. Journal of Experimental Biology, 216(7), 1338-1349. DOI: $10.1242 /$ jeb.074690 
542 Pavei, G., Seminati, E., Cazzola, D., \& Minetti, A. E. (2017). On the estimation accuracy of the

$543 \quad 3 \mathrm{D}$ body center of mass trajectory during human locomotion: inverse vs. forward

544 dynamics. Frontiers in physiology, 8, 129. DOI:

$545 \quad$ https://doi.org/10.3389/fphys.2017.00129

546 Hagio, S., \& Kouzaki, M. (2014). The flexible recruitment of muscle synergies depends on the

547

548

549

550

551

552

553

554

555

556

557

558

559

560

561

562

563

564

565

566

567

568

569

570

571

572

573

574

575

576

577

578

579

580

581

582

583

584

585

586

587 required force-generating capability. Journal of neurophysiology, 112(2), 316-327. DOI: https://doi.org/10.1152/jn.00109.2014

Hagio, S., Fukuda, M., \& Kouzaki, M. (2015). Identification of muscle synergies associated with gait transition in humans. Frontiers in human neuroscience, 9, 48. DOI: https://doi.org/10.3389/fnhum.2015.00048

Hildebrand, M. (1977). Analysis of asymmetrical gaits. Journal of Mammalogy, 58(2), 131-156. DOI: https://doi.org/10.2307/1379571

Ivanenko, Y. P., Cappellini, G., Poppele, R. E., \& Lacquaniti, F. (2008). Spatiotemporal organization of $\alpha$-motoneuron activity in the human spinal cord during different gaits and gait transitions. European Journal of Neuroscience, 27(12), 3351-3368. DOI: https://doi.org/10.1111/j.1460-9568.2008.06289.x

Lacquaniti, F., Ivanenko, Y. P., \& Zago, M., Patterned control of human locomotion, J. Physiol. 590(10) (2012), 2189-2199. DOI: https://doi.org/10.1113/jphysiol.2011.215137.

Lee, D. D. \& Seung, H. S., Learning the parts of objects by non-negative matrix factorization, Nature 401(1999) 788-791. DOI: https://doi.org/10.1038/44565

Lindstedt, S. L., Hoppeler, H. A. N. S., Bard, K. M., \& Thronson Jr, H. A. (1985). Estimate of muscle-shortening rate during locomotion. American Journal of Physiology-Regulatory, Integrative and Comparative Physiology, 249(6), R699-R703.

MacLellan, M. J., Modular organization of muscle activity patterns in the leading and trailing limbs during obstacle clearance in healthy adults, Exp. Brain Res. 235(7) (2017) 20112026. DOI: https://doi.org/10.1007/s00221-017-4946-z

Mehrabi, N., Schwartz, M. H., \& Steele, K. M., Can altered muscle synergies control unimpaired gait?, J. Biomech. 90 (2019) 84-91. https://doi.org/10.1016/j.jbiomech.2019.04.038

Minetti, A. E., The mathematical description (Lissajous contour) of the 3D trajectory of the body centre of mass: a locomotor 'signature' for the physiology, biomechanics and pathology of human and animal gaits. Gait Posture 30 (2009), S153. http://dx.doi.org/10.1016\%2Fj.gaitpost.2009.08.230

Minetti, A. E., Ardigo, L. P., Reinach, E., \& Saibene, F., The relationship between mechanical work and energy expenditure of locomotion in horses, J. Exp. Biol. 202(17) (1999) 23292338. Pubmed: http://www.ncbi.nlm.nih.gov/pubmed/10441084

Minetti, A. E., Cisotti, C. and Mian, O. S., The mathematical description of the body centre of mass 3D path in human and animal locomotion, J. Biomech 44(8) (2011) 1471-7. https://doi.org/10.1016/j.jbiomech.2011.03.014

Minetti, A. E., Pavei, G., \& Biancardi, C. M., The energetics and mechanics of level and gradient skipping: Preliminary results for a potential gait of choice in low gravity environments. Planet space sci. 74(1) (2012) 142-145. DOI: https://doi.org/10.1016/j.pss.2012.06.004.

Minetti, A. E., The biomechanics of skipping gaits: a third locomotion paradigm?, Proc. R. Soc. Lond. [Biol]. 265(1402) (1998) 1227-1233. DOI: https://doi.org/10.1098/rspb.1998.0424

Neptune, R. R., Clark, D. J., \& Kautz, S. A., Modular control of human walking: a simulation study, J. Biomech. 42(9) (2009) 1282-1287. https://doi.org/10.1016/j.jbiomech.2009.03.009

PeerJ reviewing PDF | (2020:10:54165:2:0:NEW 15 Jul 2021) 
588 Oliveira, A. S., Gizzi, L., Ketabi, S., Farina, D., \& Kersting, U. G., Modular control of treadmill $589 \quad$ vs overground running, PLoS One. 11(4) (2016), e0153307.

$590 \quad$ https://doi.org/10.1371/journal.pone.0153307

591

592

593

594

595

596

597

598

599

600

601

602

603

604

605

606

607

608

609

610

611

612

613

614

615

616

617

618

619

620

621

622

623

624

625

626

627

628

629

630

631

632

Pavei, G., Biancardi, C. M., \& Minetti, A. E., Skipping vs. running as the bipedal gait of choice in hypogravity, J. Appl. Physiol. 119(1) (2015) 93-100. DOI:

https://doi.org/10.1152/japplphysiol.01021.2014.

Pérez-Nombela, S., Barroso, F., Torricelli, D., de Los Reyes-Guzmán, A., Del-Ama, A. J., Gómez-Soriano, J., Pons \& Gil-Agudo, Á. (2017). Modular control of gait after incomplete spinal cord injury: differences between sides. Spinal Cord, 55(1), 79-86.

Saibene, F., \& Minetti, A. E., Biomechanical and physiological aspects of legged locomotion in humans, Eur. J. Appl. Physiol. 88(4-5)(2003) 297-316. DOI:

https://doi.org/10.1007/s00421-002-0654-9

Saito, A., Tomita, A., Ando, R., Watanabe, K., \& Akima, H., Muscle synergies are consistent across level and uphill treadmill running, Sci. Rep. 8(1) (2018b) 5979. DOI: https://doi.org/10.1038/s41598-018-24332-z.

Saito, A., Tomita, A., Ando, R., Watanabe, K., \& Akima, H., Similarity of muscle synergies extracted from the lower limb including the deep muscles between level and uphill treadmill walking, Gait posture, 59 (2018a) 134-139. DOI: https://doi.org/10.1016/j.gaitpost.2017.10.007

Santuz, A., Ekizos, A., Eckardt, N., Kibele, A., \& Arampatzis, A., Challenging human locomotion: stability and modular organisation in unsteady conditions, Sci. Rep. 8(1) (2018a) 2740. DOI: ht tps://doi.org/10.1038/s41598-018-21018-4

Santuz, A., Ekizos, A., Janshen, L., Mersmann, F., Bohm, S., Baltzopoulos, V. \& Arampatzis, A. Modular control of human movement during running: an open access data set. Front. Physiol., 9 (2018b). 10.3389/fphys.2018.01509.

Shuman, B. R., Goudriaan, M., Desloovere, K., Schwartz, M. H., \& Steele, K. M. (2019). Muscle synergies demonstrate only minimal changes after treatment in cerebral palsy. Journal of neuroengineering and rehabilitation, 16(1), 1-10.

Snyder, K. L., \& Farley, C. T. (2011). Energetically optimal stride frequency in running: the effects of incline and decline. Journal of Experimental Biology, 214(12), 2089-2095.

Steele, K. M., Tresch, M. C., \& Perreault, E. J. (2013). The number and choice of muscles impact the results of muscle synergy analyses. Frontiers in computational neuroscience, $7,105$.

Stegeman, D., \& Hermens, H. (2007). Standards for surface electromyography: The European project Surface EMG for non-invasive assessment of muscles (SENIAM). Enschede: Roessingh Research and Development, 108-12.

Ting, L. H., Chiel, H. J., Trumbower, R. D., Allen, J. L., McKay, J. L., Hackney, M. E. \& Kesar, T. M. Neuromechanical principles underlying movement modularity and their implications for rehabilitation, Neuron. 86(1) (2015) 38-54. http://dx.doi.org/10.1016/j.neuron.2015.02.042

Ting, L. H., Macpherson, J. M., A limited set of muscle synergies for force control during a postural task, J Neurophysiol. 93(1) (2005) 609-13. DOI: https://doi.org/10.1152/jn.00681.2004

Ting, L. H., McKay, J. L., Neuromechanics of muscle synergies for posture and movement, Curr. Opin. Neurobiol. 17(6) (2007) 622-628. DOI: https://doi.org/10.1016/j.conb.2008.01.002.

Peer] reviewing PDF | (2020:10:54165:2:0:NEW 15 Jul 2021) 
633 Torres-Oviedo, G., and Ting, L. H., Muscle synergies characterizing human postural responses, $634 \quad$ J. Neurophysiol. 98 (2007) 2144-2156. doi: 10.1152/jn.01360.2006

635 Tresch, M. C., \& Jarc, A., The case for and against muscle synergies, Curr. Opin. Neurobiol.

636

637

638

639

640

641

642

643

644

645

646

647

648

649

650

651

652

653

654

655

656

657

658

659 19(6) (2009) 601-607. DOI: https://doi.org/10.1016/j.conb.2009.09.002.

Tresch, M. C., Cheung, V. C., \& d'Avella, A., Matrix factorization algorithms for the identification of muscle synergies: evaluation on simulated and experimental data sets, J. Neurophysiol. 95(4) (2006) 2199-2212. DOI: 10.1152/jn.00222.2005.

Turpin, N. A., Uriac, S., \& Dalleau, G. (2021). How to improve the muscle synergy analysis methodology?. European Journal of Applied Physiology, 1-17.

Van Melick, N., Meddeler, B. M., Hoogeboom, T. J., Nijhuis-van der Sanden, M. W., \& van Cingel, R. E. (2017). How to determine leg dominance: The agreement between selfreported and observed performance in healthy adults. PloS one, 12(12), e0189876. DOI: 10.1371/journal.pone.0189876

Walter, R. M., \& Carrier, D. R., Ground forces applied by galloping dogs. J. Exp. Biol. 210(2) (2007) 208-216. DOI: 10.1242/jeb.02645

Whitall, J., \& Caldwell, G. E., Coordination of symmetrical and asymmetrical human gait, J. Mot. Behav. 24(4) (1992), 339-353. DOI: https://doi.org/10.1080/00222895.1992.9941630

Winter, D. A., Biomechanics and Motor Control of Human Movement, Fourth edition. John Wiley \& Sons, Inc., Toronto, 2003

Yokoyama, H., Kaneko, N., Ogawa, T., Kawashima, N., Watanabe, K., \& Nakazawa, K., Cortical Correlates of Locomotor Muscle Synergy Activation in Humans: An Electroencephalographic Decoding Study, iScience. 15 (2019) 623-639. https://doi.org/10.1016/j.isci.2019.04.008.

Yokoyama, H., Ogawa, T., Kawashima, N., Shinya, M., \& Nakazawa, K., Distinct sets of locomotor modules control the speed and modes of human locomotion, Sci. Rep, 6 (2016) 36275. DOI: https://doi.org/10.1038/srep36275 


\section{Table $\mathbf{1}$ (on next page)}

Spatiotemporal patterns of walking, running and unilateral skipping at $1.81 \mathrm{~m} \cdot \mathrm{s}^{-1}$.

In case of parametric test, ANOVA and t-test with Bonferroni correction tests were used. In case of non-parametric, Kruskal-Wallis and Wilcoxon rank-sum test (also with Bonferroni correction) was used. Significant differences between gaits were established at $p$ values $<0.05$ 


\begin{tabular}{|c|c|c|c|c|c|c|c|c|c|c|}
\hline & \multicolumn{3}{|c|}{ ANOVA (or Kruskal-Wallis) } & \multicolumn{4}{|c|}{ Gait (or leg) } & \multicolumn{3}{|c|}{ Post-hoc (Gait A vs Gait B) } \\
\hline & Parametric & $\mathrm{F}($ or $\mathrm{H})$ & $p$ & W & $\mathrm{R}$ & $\mathrm{S}_{\text {trailing }}$ & $\mathrm{S}_{\text {leading }}$ & Gait A & Gait B & $p$ \\
\hline \multirow{6}{*}{$\begin{array}{l}\text { Duty } \\
\text { Factor }\end{array}$} & \multirow{6}{*}{ False } & \multirow{6}{*}{35.98} & \multirow{6}{*}{$<0.01$} & \multirow{7}{*}{$0.58 \pm 0.02$} & \multirow{6}{*}{$0.45 \pm 0.07$} & \multirow{6}{*}{$0.28 \pm 0.06$} & \multirow{6}{*}{$0.44 \pm 0.04$} & $\mathrm{R}$ & $\mathrm{S}_{\text {trailing }}$ & 0.002 \\
\hline & & & & & & & & $\mathrm{R}$ & $\mathrm{S}_{\text {leading }}$ & 1 \\
\hline & & & & & & & & $\mathrm{R}$ & W & 0.002 \\
\hline & & & & & & & & $\mathrm{S}_{\text {trailing }}$ & $\mathrm{S}_{\text {leading }}$ & 0.001 \\
\hline & & & & & & & & $\mathrm{S}_{\text {trailing }}$ & W & $<0.001$ \\
\hline & & & & & & & & $\mathrm{S}_{\text {leading }}$ & W & $<0.001$ \\
\hline \multirow{3}{*}{$\begin{array}{c}\text { Stride } \\
\text { Frequency }(\mathrm{Hz})\end{array}$} & \multirow{3}{*}{ False } & \multirow{3}{*}{32.19} & \multirow{3}{*}{$<0.01$} & & \multirow{3}{*}{$1.32 \pm 0.08$} & \multirow{3}{*}{\multicolumn{2}{|c|}{$1.54 \pm 0.2$}} & $\mathrm{R}$ & W & $<0.001$ \\
\hline & & & & \multirow[t]{2}{*}{$1.13 \pm 0.07$} & & & & $\mathrm{~S}$ & W & $<0.001$ \\
\hline & & & & & & & & $\mathrm{R}$ & $\mathrm{S}$ & 0.001 \\
\hline \multirow{3}{*}{$\begin{array}{c}\text { Stride } \\
\text { Length (m) }\end{array}$} & \multirow{3}{*}{ True } & \multirow{3}{*}{53.79} & \multirow{3}{*}{$<0.01$} & \multirow{3}{*}{$1.6 \pm 0.09$} & \multirow{3}{*}{$1.38 \pm 0.08$} & \multirow{3}{*}{\multicolumn{2}{|c|}{$1.19 \pm 0.13$}} & $\mathrm{R}$ & $\mathrm{S}$ & 0.001 \\
\hline & & & & & & & & $\mathrm{R}$ & W & $<0.001$ \\
\hline & & & & & & & & S & W & $<0.001$ \\
\hline $\begin{array}{c}\text { Flight Time } \\
\text { (fraction) }\end{array}$ & True & - & - & - & $0.17 \pm 0.07$ & 0.31 & 0.07 & S & $\mathrm{R}$ & $<0.001$ \\
\hline
\end{tabular}


Figure 1

EMG envelopes

Averaged $(n=14)$ electromyographic linear envelopes of 7 recorded muscles for each gait as a function of the gait cycle at $1.81 \mathrm{~m} \cdot \mathrm{s}^{-1}$. For each subject, linear envelope from each muscle were previously normalized by the peak throughout 20 cycles. Muscle abbreviations:

Gastrocnemius Medius (Gas), Soleus (Sol), Rectus Femoris (RF), Vastus Medialis (Vas), Tibialis anterior (Tib), Biceps Femoris (BF) and Gluteus Medius (GluM). A.U., arbitrary unit.

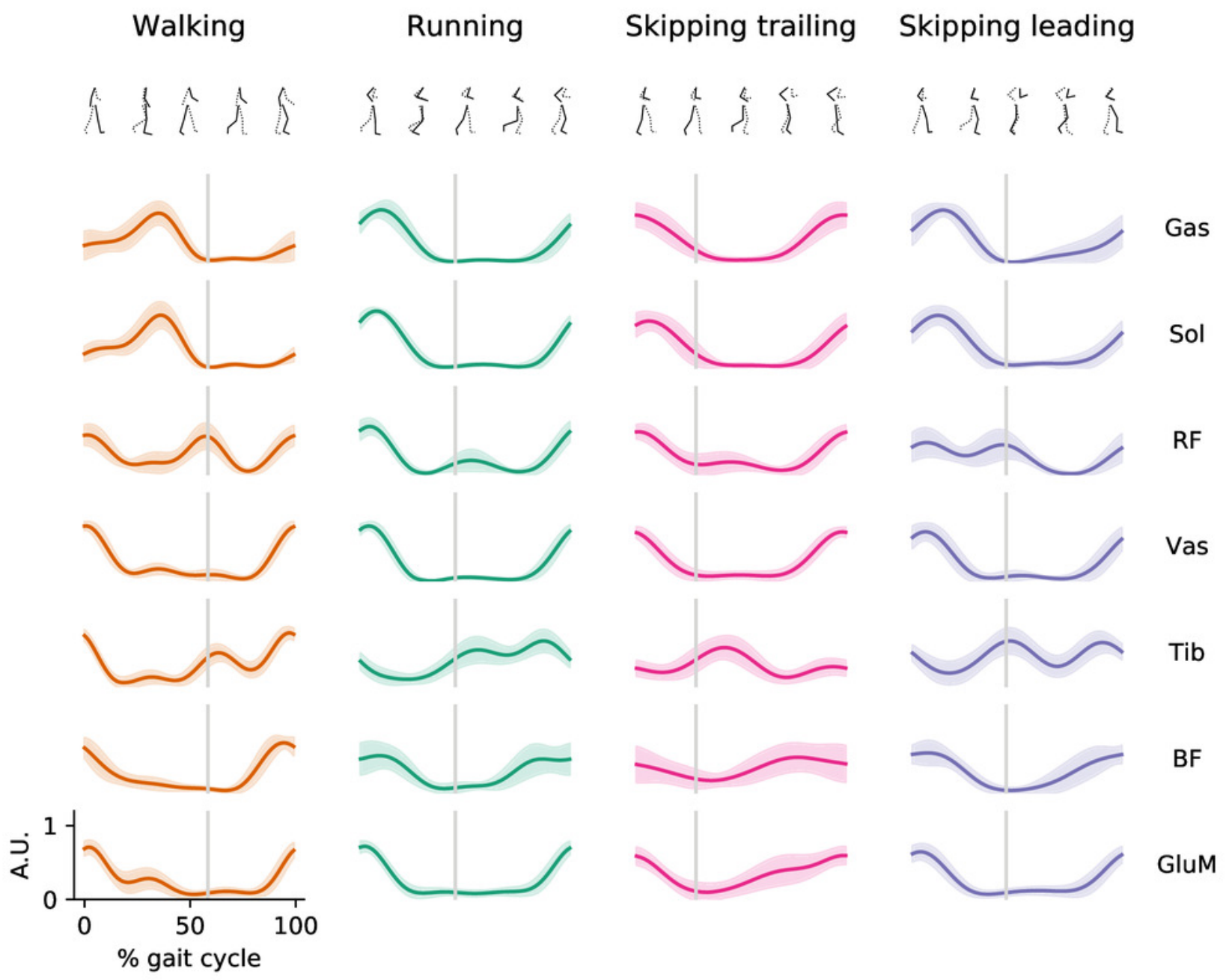




\section{Figure 2}

Number of synergies

Number of synergies (mean $\pm \mathrm{s} . \mathrm{d}$.) between gaits at $1.81 \mathrm{~m} \cdot \mathrm{s}^{-1}$. No significant differences were found $(p=0.07)$.

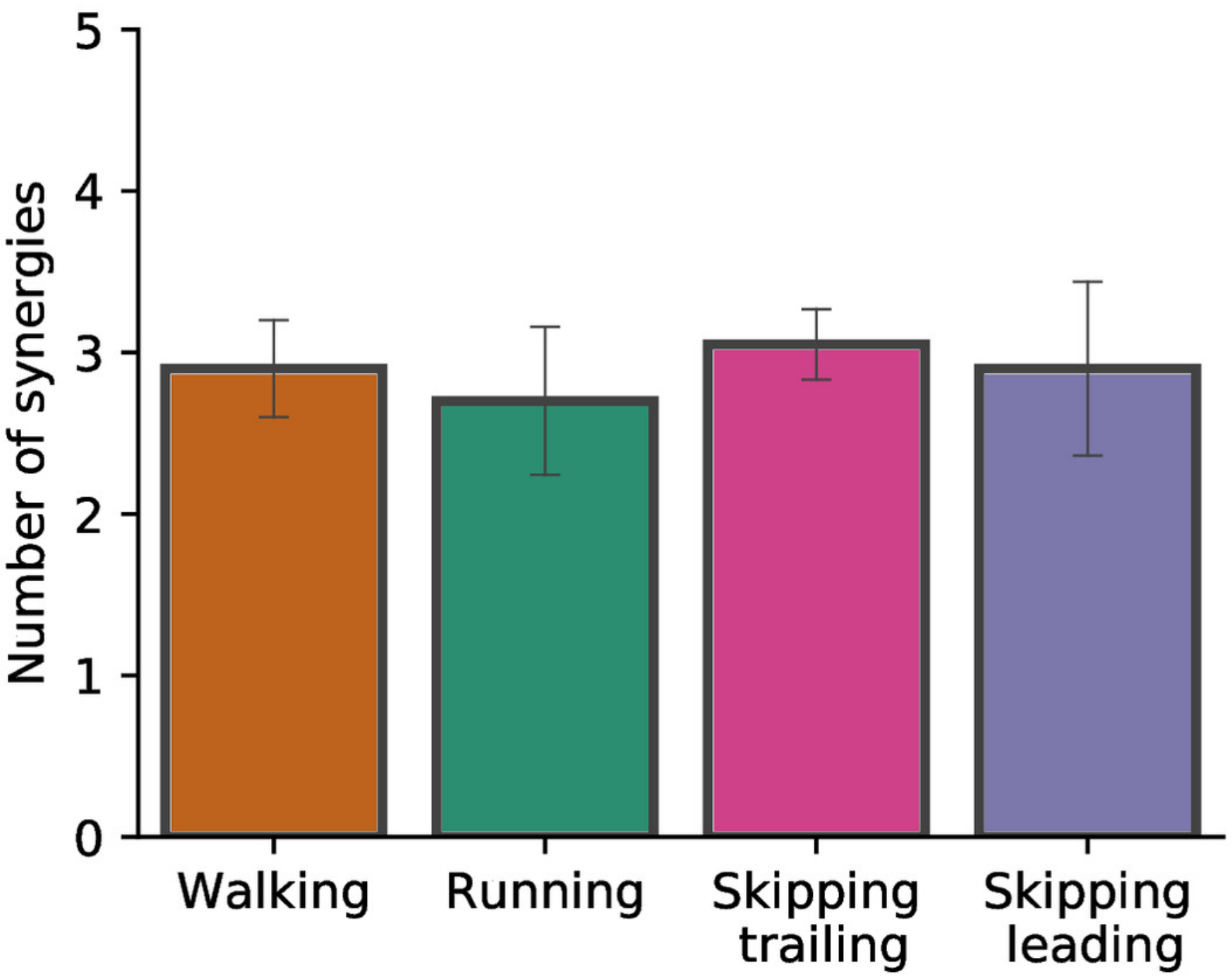


Figure 3

Activation timings profiles and muscle synergies of walking, running and skipping (both legs) at $1.81 \mathrm{~m} \cdot \mathrm{s}^{-1}$.

Solid lines in activation timing profiles indicate average $(n=14)$ and shaded area represent \pm s.d. Vertical lines divide the stance (on left) and swing (on right) phases. Muscle weightings are represented by bars plot. Muscle abbreviations: Gastrocnemius Medius (Gas), Soleus (Sol), Rectus Femoris (RF), Vastus Medialis (Vas), Tibialis anterior (Tib), Biceps Femoris (BF) and Gluteus Medius (GluM).
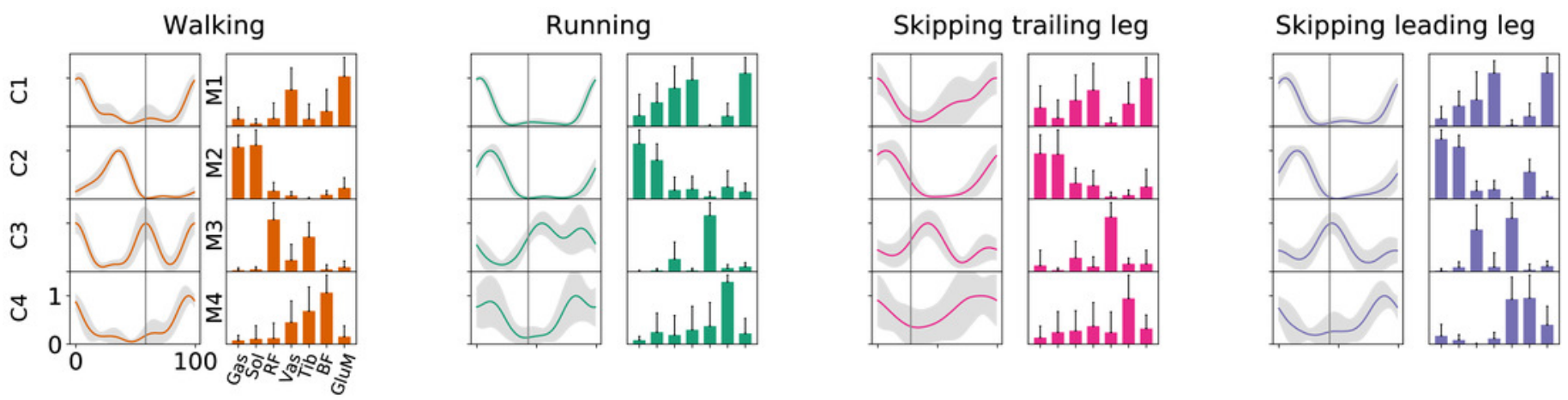
Figure 4

Synergy similarities between gaits at $1.81 \mathrm{~m} \cdot \mathrm{s}^{-1}$.

Cosine similarities (cosim) means $(n=14) \pm$ s.d across gaits comparisons. * indicate synergies that do not reach the similarity threshold.
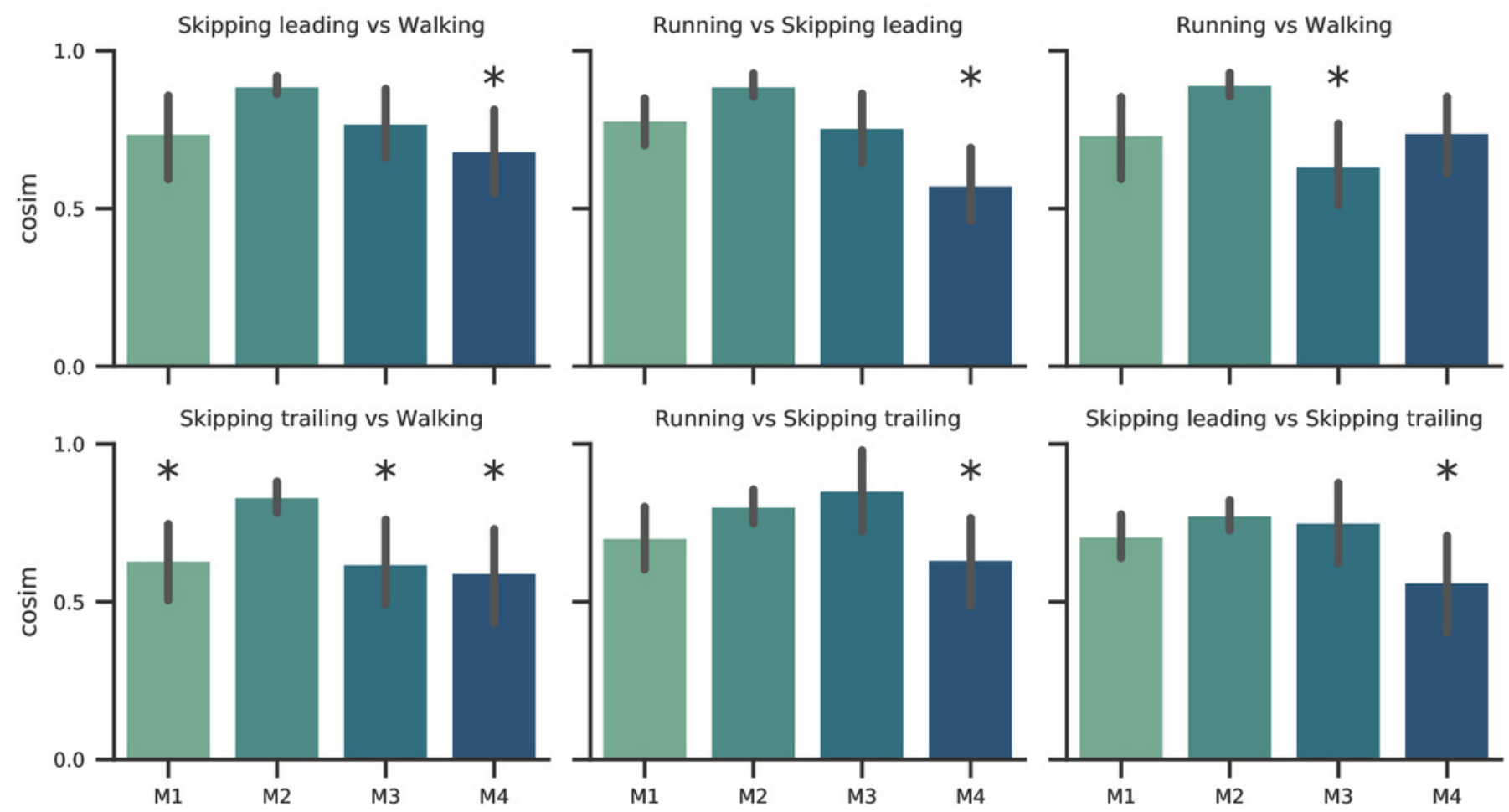
Figure 5

Centers of activation in different gaits at $1.81 \mathrm{~m} \cdot \mathrm{s}^{-1}$.

Averaged ( $n=14)$ centers of activation (COA) \pm s.d. of each motor module plotted in polar coordinates. Significant differences between gaits are indicated above each plot.

Abbreviations: Running (R), trailing leg in skipping (Str), leading leg in skipping (Slead) and walking (W).

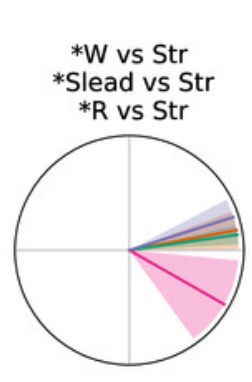

C1

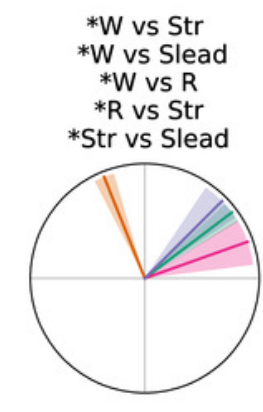

$\mathrm{C} 2$

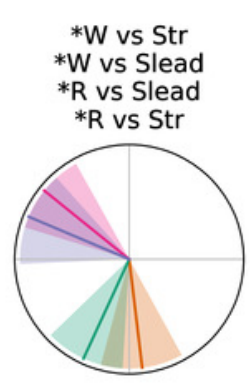

C3

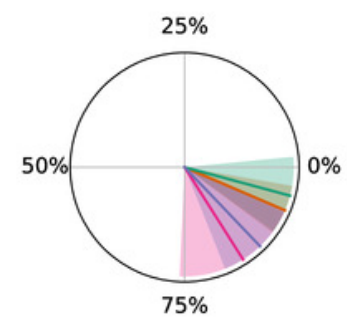

C4 
Figure 6

Trajectories of the center of mass in local coordinates of one representative subject at $1.81 \mathrm{~m} \cdot \mathrm{s}^{-1}$.

Each closed loop corresponds to one stride of walking (A), running (B) and skipping (C), at different scale. The $x$ axis indicates progression direction. Blue: weight acceptance (synergy module 1); Red: propulsion (synergy module 2); Violet: modules $1 \& 2$ in leading skipping; Yellow: pendular transference in walking; Green: ballistic phase in running and skipping.

A

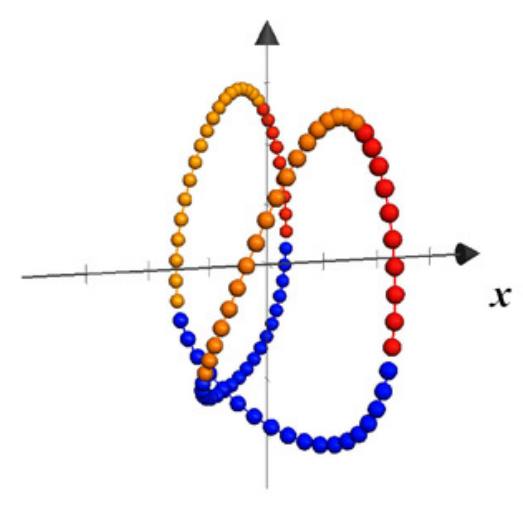

B

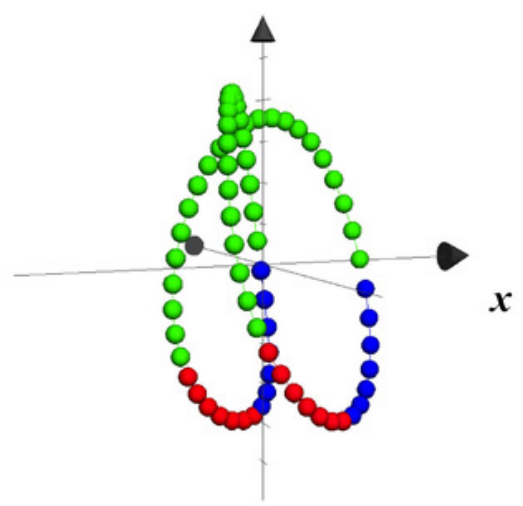

C

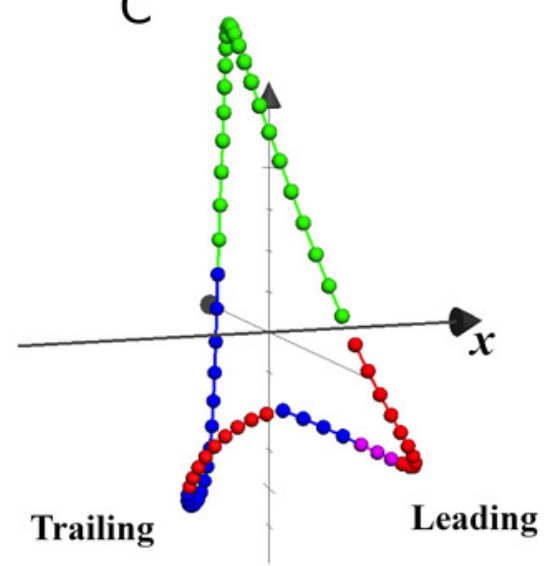

